\title{
LAND TITLES ACT CASE LIST
}

Institute of Law Research and Reform

This list of cases was prepared for the internal use of the Institute of Law Research and Reform in its project on the revision of the Land Titles Act. It seems likely however to prove a useful reference for practitioners and others doing research into this area of the law. It was prepared during the summer of 1974 under the direction of Professor M. Sychuk of the Faculty of Law, University of Alberta, by Heather Lamoureux and Norman Picard who at the time were students in the Faculty of Law. The list was recently updated to include cases decided up to February of 1976. 
Section 2 Re MacKenzie \& Mann Ltd. and Foley (1909) 10 W.L.R. 668(Sask.).

Re Registration of a Transfer of Coal Rights (1914) 7 W.W.R. 769 (Sask.).

Friesen v. Great West Permanent Loan Co.; Mennonite Land Sales Co. Ltd. v. Friesen [1924] 3 W.W.R. 883 (Sask.).

In Re Land Titles Act; In Re Fielding and North of Scotland Mtge. Co.[1927] 2W.W.R. 623(Alta.).

Knight Sugar Co. Ltd. v. Alberta Railway and Irrigation Co. [1938] 1 W.W.R. 234 (Alta.).

Landowners Mutual Minerals Ltd. v. Registrar of Land Titles (1952) 6 W.W.R. (NS) 230 (Sask.).

Re Quieting Titles Act; Re a Certain Lot New Westminster District (1962) 40 W.W.R. (NS) 182 (B.C.).

Central Mortgage and Housing Corporation v. Adams (1968) 67 W.W.R. (NS) 561 (B.C.).

Section 19 Re Land Registry Act; Re Continental Explosives (1964) 49 W.W.R. (NS) 762 (B.C.).

Section 20 In Re Bentley and Morris (1892)12 Can. Law Times 119.

Greenshields Ltd. and Ritchie(1905)6Terr. L.R. 208.

Re American-Abell Engine \& Thresher Co. and Noble (1906)6 Terr. L.R. 359.

Gorman and Co. v. Archibald (1908) 1 A.L.R. 525.

Re Land Titles Act (1912)5 A.L.R. 385.

Re Toth and J. I. Case Threshing Machine Co. (1910) 14 W.L.R. 704 (Sask.).

Hall v. Registrar of Yorkton Land Registration District (1911) 16 W.L.R. 568(Sask.).

Re Bank of Hamilton and McAllister (1912) 22 W.L.R. 849 (Alta.).

Re Sinclair (1913) 23W.L.R. 286(Alta.).

Siemens v. Dirks (1913) 25 W.L.R. 633(Man.).

Section 25 Siemens v. Dirks (1913) 25 W.L.R. 633(Man.).

Friesen v. Great West Permanent Loan Co.; Mennonite Land Sales Co. Ltd. v. Friesen [1924] 3 W.W.R. 883 (Sask.).

In Re Land Registry Act; In Re Bankruptcy Act; In Re Chambers [1943] 1W.W.R. 297(B.C.).

Summers' Agricultural Services Ltd. v. Vacationland Properties Ltd.[1972] 2W.W.R. 477(Alta.).

Section 34 Re Land Titles Act (1913) 7 A.L.R. 32.

Re Canadian Explosives and Land Registry Act [1918] 1 W.W.R. 399(B.C.). 
In Re Land Titles Act; Olson Lease [1920] 1 W.W.R. 739 (Sask.).

Bremner v. Trusts \& Guarantee Co. [1928] 3 W.W.R. 415(Alta.).

Section 38 Re Land Registry Act and Shaw (1915) 8 W.W.R. 1270 (B.C.).

Section 51 St. Germain v. Reneault (1909) 2 A.L.R. 371.

Re Roueche (1907) 7 W.L.R. 278(Alta.).

St. Germain v. Reneault (1910) 12W.L.R. 169 (Alta.).

Re Discharge of Mortgage (1913) 5W.W.R. 126(Sask.).

Re Registration of Mortgage (1913) 5W.W.R.1189(Sask.).

Re Land Titles Act and Allan and O'Connor [1918] 1 W.W.R. 440(Alta.).

Gillson v. Gillson (1961) 36 W.W.R. (NS) 480 (B.C.).

Section 52 In Re Land Titles Act, Sec. 51; Hyndman v. City of Edmonton [1949] 1 W.W.R. 751 (Alta.).

Bank of Montreal v. University of Saskatchewan (1953) 9W.W.R.(NS) 193 (Sask.).

In Re S. 51 Land Titles Act (Grieve's Application) (1953) 10W.W.R. (NS) 49 (Alta.).

Re Land Titles Act, S. 52; Re Certain Restrictive Covenants (1963) 44 W.W.R. (NS) 480 (Alta.).

Marahens and Marahens v. Oluk [1975] 1 W.W.R. 137 (Alta.).

Section 54 In Re Land Titles Act (1899) 4 Terr. L.R. 300.

Greenshields Ltd. and Ritchie (1905)6 Terr. L.R. 208.

Re American-Abell Engine \& Thresher Co. and Noble (1906)6 Terr. L.R. 359.

In Re Gordon (1901) 7 Terr. L.R. 134.

Devenish v. Connacher (1930) 24 A.L.R. 535.

Re International Harvester Co. of America (1909) 11 W.L.R. 29(Sask.).

Re Land Titles Act (No.2)(1913) 24 W.L.R. 385(Alta.).

Re Gardiner and District Registrar at Nelson (1914) 27 W.L.R. 536(B.C.).

Re Land Titles Act (1913) 4 W.W.R. 677(Alta.).

Re Land Titles Act (1913) 4 W.W.R. 857 (Sask.).

Arnold v. National Trust Co.; Re Land Titles Act (1913) 4W.W.R.1113(Alta.).

Re Registration of Mortgage (1914) 5W.W.R. 1189(Sask.).

Re C.P.R. Co. and Reid(1914)6 W.W.R.1160(Sask.).

Re Registration of a Transfer of Mortgage (1915) 9 W.W.R. 491 (Sask.).

Re Land Titles Act etc. [1918] 1 W.W.R. 411 (Sask.).

Re Land Titles Act [1918] 2W.W.R.939(Sask.). 
Re Land Titles Act; Yorkton Gas Co. Ltd.'s Case [1918] 3W.W.R. 15(Sask.).

Re Land Titles Act [1918] 3W.W.R. 348(Sask.).

In Re Land Titles Act; Weightman's Case [1919] 1 W.W.R. 44 (Sask.).

In Re Land Titles Act [1919] 1 W.W.R. 628(Sask.).

Home Oil Distributors v. Bennett [1936] 1 W.W.R. 385 (B.C.).

In Re Land Registry Act; In Re Conveyance from Cawley to Lumley[1947]2 W.W.R. 526(B.C.).

Re Land Registry Act; Re Application A3554 (1967) 60 W.W.R.(NS) 509 (B.C.).

Re Land Registry Act; Re Beeler's Application (1970) 71 W.W.R.(NS) 680 (B.C.).

Section 55 Reichert v. Registrar for Regina Land Registration District (1965) 53 W.W.R. (NS) 382 (Sask.).

Section 56 In Re Kerr(1902) 5Terr. L.R. 297.

Tucker v. Armour (1907) 6 W.L.R. 93.

Smith v. Smith (1915) 8W.W.R. 1077 (Alta.).

In Re Church (1923) 3 W.W.R. 405(Alta.).

In Re Land Titles Act (Ancey's Case) (1924) 3 W.W.R. 506 (Sask.).

Lapin v. Abigail[1934] 3W.W.R. 689(Aust.).

Knight Sugar Co. Ltd. v. Alberta Railway and Irrigation Co. [1938] 1 W.W.R. 234 (Alta.).

L. \& C. Lumber Co. Ltd. v. Lundgren [1942] 3 W.W.R. 557 (B.C.).

Davidson v. Davidson [1946] 2D.L.R. 289(B.C.).

Hutton v. Holt [1950] 2W.W.R. 17(Alta.).

Forrest v. Howe (1951) 4 W.W.R. (NS) 300 (B.C.).

Re McCurdy Supply Co. Ltd. v. Doyle; Russell and Community Investments Ltd. (1956) 20 W.W.R. (NS) 125 (Man.).

Stonehouse v. A.G. of British Columbia (1962) 37 W.W.R. (NS) 62 (B.C.).

Re Land Registry Act; Heller v. Registrar, Vancouver Land Registration District (1963) 41 W.W.R. (NS) 641 (B.C.).

Gillson v. Gillson (1961) 36 W.W.R. (NS) 480(B.C.).

Kaup v. Imperial Oil Limited (1962) 37 W.W.R. (NS) 193 (Sask.).

Protective Holdings Ltd. v. $M$ \& P Transport Ltd. (1969) 70 W.W.R. (NS) 591 (Alta.).

Progressive Services Ltd. and Vancouver Boat Shop Ltd. v. Corporation of District of Burnaby (1970) 75 W.W.R. (NS) 303(B.C.). 
Section 57 In Re Bentley and Morris (1892) 12 Can. Law Times 119. Grace v. Kuebler (1917) 12 A.L.R. 392.

Mackie v. Standard Trusts Company (1922) 17 A.L.R. 236. Robinson v. Ford (1914) 31 W.L.R. 13 (Sask.).

Morris v. Morris and French [1931] 3 W.W.R. 427 (B.C.).

Section 58 McRae v. Planta [1924] 2 W.W.R. 323 (B.C.).

Section 61 Glenn v. Scott et al. (1898) 2 Terr. L.R. 339.

In Re MacDonald Estate (1925) 21 A.L.R. 66.

Short v. Graham (1908) 7 W.L.R. (Alta.).

Reeves v. Konschur (1909) 10 W.L.R. 680 (Sask.).

Colonial Investment and Loan Co. v. Foisie (1911) 19 W.L.R. 748 (Sask.).

Ross and Phillips v. Schmitz (1913) 5 W.W.R. 399 (Sask.). Evans et al. v. Ashcroft et al. (1915) 8 W.W.R. 899 (Alta).

Montreal Trust Co. v. Boggs and Beresford (1915) 8 W.W.R. 1200 (Sask.).

Great West Lumber Co. v. Murrin and Gray [1917] 1 W.W.R. 945 (Alta.).

Dominion of Canada Investment and Debenture Co. Ltd. v. Carstens [1917] 3 W.W.R. 153 (Sask.).

Re L.T.A. and Re Ronald and Summers [1917] 3 W.W.R. 626 (Alta.).

Trusts and Guarantee Co. Ltd. v. Stephens [1919] 3 W.W.R. 410 (Alta.).

Trusts \& Guarantee Co. Ltd. v. Landreville and Singer [1922] 2 W.W.R. 586 (Alta.).

Walsh v. Popham [1924] 2 W.W.R. 1193 (Alta.).

Trusts \& Guarantee Co. Ltd. v. Monk [1925] 1 W.W.R. 5 (Alta.).

In Re MacDonald Estate [1925] 1 W.W.R. 1031 (Alta.).

Sanford v. Frizzle and Elliot [1925] 2 W.W.R. 601 (Alta.).

Anderson v. Stasiuk [1927] 1 W.W.R. 49 (Sask.).

Trusts and Guarantee Co. Ltd. v. McLeod and Buxton [1928] 3 W.W.R. 205 (Alta.).

Sokolov v. Kachmark [1929] 1 W.W.R. 353 (Man.).

Sakaliuk v. Corry [1930] 1 W.W.R. 424 (Alta.).

Devenish v. Connacher [1930] 2 W.W.R. 254 (Alta.).

Hamlyn v. Kaplan [1936] 2 W.W.R. 293 (Alta.).

Superior Builders v. Scott and Shore [1937] 2 W.W.R. 274 (Man.).

Pollock v. Shapera [1938] 1 W.W.R. 310 (Man.).

Pfeifle v. Bachinsky et al. [1939] 2 W.W.R. 389 (Alta.).

In Re Forster Estate [1941] 3 W.W.R. 449 (Alta.).

Central Mortgage and Housing Corporation v. Ward (1957-58) 23 W.W.R. 319 (Alta.). 
Central Mortgage and Housing Corporation v. Conaty (1967) 59 W.W.R. (NS) 11 (Alta.).

Central Mortgage and Housing Corporation v. Johnson and Chalazan [1971] 5 W.W.R. 163 (Sask.).

Section 62 Great West Lumber Co. v. Murrin \& Gray (1916) 11 A.L.R. 173.

Section 63 Canada Permanent Mortgage Corporation v. Natha Singh [1921] 2 W.W.R. 361 (B.C.).

Gutschenritter v. Ball [1923] 3 W.W.R. 619 (Sask.).

Sakaliuk v. Corry [1930] 1 W.W.R. 424 (Alta.).

Majestic Mines Ltd. v. A.G. for Alberta [1941] 2 W.W.R. 353 (Alta.).

Essery v. Essery; Tatko v. Leifke [1947] 2 W.W.R. 1044 (Alta.).

Boyczuk v. Perry and Perry [1948] 1 W. W.R. 495 (Alta.).

In Re Land Titles Act and a Certain Certificate of Title (1952) 7 W.W.R. (NS) 21 (Sask.).

In Re Land Titles Act and Yukon Oils Ltd. (1952) 7 W.W.R. (NS) 47 (Alta.).

In Re Mechanics' Lien Act; United Sheet Metal Ltd. v. Hager (1953) 7 W.W.R. (NS) 481 (Alta.).

Balzer and Balzer v. Registrar of Moosomin Land Registration District (1954) 11 W.W.R. (NS) 469 (Sask.).

C.P.R. v. Turta (1954) 12 W.W.R. (NS) 97 (Alta.).

Henderson and Henderson v. Montreal Trust Co. (Administrator of Murphy Estate) (1955) 14 W.W.R. (NS) 210 (Alta.).

Imperial Oil Ltd. v. Conroy and Berthiaume (Judicial Trustee of Gratrix Estate) (1954) 12 W.W.R. (NS) 569 (Alta.).

Carr \& Son and Carr Plumbing \& Heating Ltd. v. Rayward and Bell (1955) 17 W.W.R. (NS) 399 (B.C.).

Lukiv v. Abbotsford School District No. 34 (1958) 26 W.W.R. (NS) 645 (B.C.).

Stonehouse v. A.G. of British Columbia (1962) 37 W.W.R. (NS) 62 (B.C.).

Fellger v. Canada Trust Co. (1961) 34 W.W.R. (NS) 418 (Alta.).

Kaup v. Imperial Oil Limited (1962) 37 W.W.R. (NS) 193 (Sask.).

Credit Foncier Franco-Canadien v. Bennett and Allen and A.G. for British Columbia (1963) 43 W.W.R. (NS) 545 (B.C.) (re costs: (1964) 47 W.W.R. (NS) 369).

Duncan v. Joslin (1965) 51 W.W.R. (NS) 346 (Alta.).

Hudson's Bay Co. v. Shivak (1965) 52 W.W.R. (NS) 695 (Sask.).

Re Land Registry Act; Canada Permanent Mortgage Corp. v. Registrar of Titles (1966) 58 W.W.R. (NS) 9 (B.C.). 
Re Quieting Titles Act; Re a Certain Lot, Kootenay District (1967) 60 W.W.R. (NS) 119 (B.C.).

Public Trustee for Alberta v. Pylypow [1973] 6 W.W.R. 673 (Alta.).

Section 64 Daly v. Robertson (1892) 1 Terr. L.R. 427.

Norris v. Bentley (1895) 2 Terr. L.R. 253.

Grand Trunk Ry. Co. v. Vincent (1909) 2 A.L.R. 393.

Lindsay v. Gibboney (1931) 25 A.L.R. 347.

First National Investment Co. v. Thoroddur Oddson and Leifur Oddson [1919] 3 W.W.R. 591 (Man.).

Sorenson v. Young [1920] 1 W.W.R. 189 (B.C.)

White v. Municipal District of Inga and Pidgeon [1929] 1 W.W.R. 172 (Alta.).

Lindsay v. Gibboney [1931] 1 W.W.R. 728 (Alta.).

Manitoba Farm Loans Association v. Chapple [1944] 2 W.W.R. 282 (Man.).

Bell and Bell v. Fullerton [1945] 3 W.W.R. 77 (Sask.).

Trotzuk v. Zilka [1946] 3 W.W.R. 442 (Sask.).

Rystephaniuk v. Prosken (1951) 3 W.W.R. (NS) 76 (Man.).

Canadian Superior Oil of California and Hiebert v. District Registrar of Land Titles, District of Portage la Prairie (1953) 8 W.W.R. (NS) 417 (Man.).

Huggard Assets Ltd. v. A.G. for Alta., Minister of Lands and Mines for Alta., and A.G. of Canada (1953) 8 W.W.R. (NS) 561 (Alta.).

Reference Re Title to Mines and Minerals in S.W. $1 / 4$ of 14-53-26 W. 4th M. (1954) 13 W.W.R. (NS) 58 (Alta.).

Wardle v. Manitoba Farm Loans Association and Government of Manitoba (1955) 14 W.W.R. (NS) 289.

Re Prudential Trust Co. Ltd. and Registrar of Humboldt Land Registration District (1956) 18 W.W.R. (NS) 1 (Sask.).

Beltz v. Calgary (City), Tiran Investments Ltd. and Steinberg (1960) 31 W.W.R. (NS) 134 (Alta.).

Re Land Titles Act; Re Director of Soldier Settlement (1960) 31 W.W.R. (NS) 647 (Alta.).

Stall and Stall v. Yarosz and Yarosz (1964) 47 W.W.R. (NS) 113 (Man.).

Niebe v. Enns [1971] 3 W.W.R. 469 (Man.).

Farm Credit Corporation v. Kolotylo [1973] 2 W.W.R. 766 (Alta.).

Dukart v. Corporation of the District of Surrey and Registrar of New Westminster Land Registration District [1974] 2 W.W.R. 402 (B.C.).

Section 65 Burden v. Registrar of Land Titles for N. Alberta Land Registration District (1913) 5 W.W.R. 122 (Alta.).

Davidson v. Davidson [1946] 2 D.L.R. 289 (B.C.). 
Essery v. Essery; Tatko v. Leifke [1947] 2 W.W.R. 1044 (Alta.).

In Re Land Titles Act and A Certain Certificate of Title (1952) 7 W.W.R. (NS) 21 (Sask.).

In Re Mechanics' Lien Act; United Sheet Metal Ltd. v. Hager (1953) 7 W.W.R. (NS) 481 (Alta.).

C.P.R. v. Turta (1954) 12 W.W.R. (NS) 97 (Alta.).

Re Certain Mineral Rights; Re Land Titles Act, Sec. 82; A.G. Can. v. Toth (1959) 27 W.W.R. (NS) 230 (Sask.).

Fellger v. Canada Trust Co. (1961) 34 W.W.R. (NS) 418 (Alta.).

Kaup v. Imperial Oil Ltd. (1962) 37 W.W.R. (NS) 193 (Sask.).

Hudson's Bay Co. v. Shivak (1965) 52 W.W.R. (NS) 695 (Sask.).

Moore v. Moore (1971) 16 D.L.R. (3d) 174 (B.C.).

Section 67 Neider v. Carda of Peace River District Ltd. [1971] 2 W.W.R. 379 (Alta.).

Grier v. Krivak [1973] 1 W.W.R. 739 (Man.).

Section 68 In Re Land Titles Act (1899) 4 Terr. L.R. 300.

Section 70 Re Registration of Easements (1915) 8 W.W.R. 171 (Sask.). In Re Land Registry Act; In Re Harrie; City of Vancouver v. Registrar of Titles [1945] 2 W.W.R. 348 (B.C.).

In Re Land Titles Act; in Re Interprovincial Pipe Line Co. (1950) 1 W.W.R. (NS) (Sask.).

Section 73 Brogden v. Brogden (1920) 15 A.L.R. 499.

Re Anderton (1908) 8 W.L.R. 319 (Alta.).

Harris v. Keith (1911) 16 W.L.R. 433 (Alta.).

Wallace v. Potter (1913) 24 W.L.R. 262 (Alta.).

Wallace v. Potter (No. 2) (1913) 4 W.W.R. 738 (Alta.).

Sinclair v. McLellan [1919] 2 W.W.R. 782 (Alta.).

Burch v. Towder [1923] 1 W.W.R. 180 (B.C.).

Gunn v. McInnes [1923] 1 W.W.R. 353 (Man.).

Washington and Great Northern Townsite Co. v. Holbrook [1924] 1 W.W.R. 511 (B.C.).

Shirtcliffe v. Lemon [1924] 1 W.W.R. 1059 (Alta.).

Dobek v. Jennings [1928] 1 W.W.R. 348 (Alta.).

Nugent v. Moore [1938] 2 W.W.R. 561 (Man.).

Saturley v. Young [1945] 3 W.W.R. 110 (Alta.).

Boyczuk v. Perry and Perry [1948] 1 W.W.R. 495 (Alta.).

In Re Land Titles Act, Limitation of Actions Act and Dower Act; Jameson v. Hyslop [1950] 2 W.W.R. 1273 (Alta.). 
Lukiv v. Abbotsford School District No. 34 (1934) 26 W.W.R. (NS) 645 (B.C.).

Drummond v. Drummond (1965) 50 W.W.R. (NS) 538 (B.C.).

Montreal Trust Co. v. Murphy (1966) 58 W.W.R. (NS) 430 (Sask.).

Section 78 Re Location Plans (1913) 5 W.W.R. 413 (Sask.).

Section 82 Re New Westminster Sub-division (1914) 5 W.W.R. 1253 (B.C.).

Rowland v. City of Edmonton (1915) 8 W.W.R. 20 (Alta.).

Summer v. McIntosh [1918] 2 W.W.R. 293 (Sask.).

Section 84 In Re Capital Grocers Ltd. and Registrar of Land Titles (1952) 7 W.W.R. (NS) 315 (Sask.).

Re Land Registry Act; Re Approval of a Subdivision Plan (Delta District) (1961) 34 W.W.R. (NS) 41 (B.C.).

Section 85 Sheppard v. Godfrey (1915) 9 A.L.R. 416.

Section 86 In Re Land Titles Act and Yukon Oils Ltd. (1952) 7 W.W.R. (NS) 47 (Alta.).

Re Land Registry Act; Re Atlas Copco Canada Ltd. (1956) 20 W.W.R. (NS) 261 (B.C.).

Section 88 Veilleux v. The Boulevard Heights (1914) 8 A.L.R. 16.

Abbott v. Ridgeway Park, Ltd. (1915) 7 W.W.R. 1280 (Alta.).

Sheppard v. Godfrey (1915) 8 W.W.R. 999 (Alta.).

Section 89 Re Application of the Moose Jaw Securities, Ltd. (1912) 3 W.W.R. 216 (Sask.).

Re Land Titles Act and Plan I (1913) 3 W.W.R. 1032 (Sask.).

Section 97 Re Land Titles Act; Re Northern Crown Bank [1918] 1 W.W.R. 421 (Sask.).

Re Land Titles Act (1921) 2 W.W.R. 841 (Sask.).

Broersma v. Maier (1969) 72 W.W.R. (NS) 153 (Alta.).

Section 98 Tefler Bros. v. Fisher (1910) 15 W.L.R. 400 (Alta.). Roberts v. McMannis [1933] 1 W.W.R. 193 (Sask.).

Section 99 Warner v. Linahan (1919) 14 A.L.R. 433.

Re Tucker and Armour (1906) 3 W.L.R. 394. 
Section 100 Toronto Dominion Bank v. City of Saskatoon (1967) 60 W.W.R. (NS) 360 (Sask.).

Section 104 Re North-West Telephone Co. (1910) 12 W.L.R. 300 (Sask.).

Re Rumely Co. and Registrar of Saskatoon Land Registration District (1912) 17 W.L.R. 160 (Sask.).

Shore v. Webber (1913) 24 W.L.R. 343 (Sask.).

Re Registration of Mortgage (1914) 5 W.W.R. 1189 (Sask.). Massey-Harris Co. Ltd. v. McDiarmid [1921] 3 W.W.R. 127 (Sask.).

Wilson v. Ward [1929] 2 W.W.R. 122 (Alta.).

Devenish v. Connacher [1930] 2 W.W.R. 254 (Alta.).

Re Judgments Act; Regina v. Hamilton (1962) 39 W.W.R. (NS) 545 (Man.).

Re Land Registry Act; Re Clary and Hassell Developments Ltd.'s Registration Application (1963) 45 W.W.R. (NS) 389 (B.C.).

Section 105 Re Spokane and Eastern Trust Co.'s Mortgage (1910) 15 W.L.R. 637 (Alta.).

Ferris v. Nowitskey (1951) 3 W.W.R. (NS) 49 (Alta.).

Re Preston (1969) 4 D.L.R. (3d) 431 (Sask.).

Section 106 In Re Tax Recovery Act; In re Banque Canadienne Nationale and Waterloo Machinery (Alta.) Ltd. [1947] 1 W.W.R. 910 (Alta.).

Barker v. Belzberg (1951) 4 W.W.R. (NS) 304 (Alta.).

Section 108 Morguard Mortgage Investments Limited v. Faro Development Corporation et al. [1975] 1 W.W.R. 737 (Alta.).

Section 109 Security Trust Co. Ltd. v. National Trust Co. Ltd. [1928] 1 W.W.R. 687 (Alta.).

Section 110 Re Land Titles Act; Re Arrears of Taxes Act [1918] 2 W.W.R. 938 (Sask.).

In Re Land Titles Act; In Re Windover and Great West Life Assurance Co.; In Re Asseltine [1927] 2 W.W.R. 414 (Alta.).

Hungerbuhler and Manuel v. Maryland [1946] 1 W.W.R. 419 (Alta.).

In Re Land Titles Act; In Re Swanston [1946] 3 W.W.R. 123 (Sask.).

Foxon v. Sandstrom [1947] 1 W.W.R. 529 (Sask.).

Moreau v. Baker and Moret [1947] 1 W.W.R. 1098 (Alta.). In Re Tone Mortgage (1954) 11 W.W.R. (NS) 646 (Sask.). In Re Land Titles Act; In Re Hadwin (1954) 12 W.W.R. (NS) 414 (Sask.). 
Puhacz (Pukacz) v. Wyrzykowski (1966) 59 W.W.R. (NS) 180 (Man.).

Re Simpson Estate (1970) 75 W.W.R. (NS) 736 (B.C.).

Loose v. Spruce Holdings and Investments Ltd. (1972) 23 D.L.R. (3d) 204 (Alta.).

Section 112 Devenish and Devenish v. Connacher [1929] 3 W.W.R. 355 (Alta.).

Gallagher v. Freeholders Oil Company Limited (1962) 40 W.W.R. (NS) 35 (Sask.).

Section 114 Re Land Registry Act; Re Mandeville et al. [1917] 1 W.W.R. 1522 (B.C.).

Re McCurdy Supply Co. Ltd. v. Doyle; Russell and Community Investments Ltd. (1956) 20 W.W.R. (NS) 125 (Man.).

Section 118 Rollefson Bros. Co. v. Olson and Mutual Life Assurance (1915) 8 W.W.R. 481 (Sask.).

Hyde v. Chapin \& Co. (1915) 8 W.W.R. 820 (Alta.).

Matthewson Bros. and Mather v. Good [1927] 1 W.W.R. 728 (Sask.).

Incorporated Synod of Diocese of Huron v. Board of Trustees of Lethbridge Northern Irrigation District [1930] 2 W.W.R. 503 (Alta.).

Section 120 B.C. Glass and Lumber Ltd. v. Kamlo Motels Ltd. and Levitsky (1961) 36 W.W.R. (NS) 429 (B.C.).

Section 122 Re Land Titles Act; Re Carrogher et al. (1913) 3 W.W.R. 1034 (Sask.).

In Re Land Titles Act; Royal Trust Co.'s Case [1921] 3 W.W.R. 246 (Sask.).

Re Land Registry Act; Re Rode's Petition (1968) 64 W.W.R. (NS) 430 (B.C.).

Section 125 St. Germain v. Reneault (1910) 12 W.L.R. 169 (Alta.).

Re Administration De Bonis Non (1914) 5 W.W.R. 1328 (Sask.).

Western Trust Co. v. Olson [1918] 3 W.W.R. 811 (Sask.).

Bremner v. Trusts \& Guarantee Co. [1928] 3 W.W.R. 415 (Alta.).

Section 128 Re Claxton (1890) 1 Terr. L.R. 283.

Re Rivers (1893) 1 Terr. L.R. 464.

Wilkie v. Jellett (1895) 2 Terr. L.R. 133 (aff'd 26 S.C.R. 282).

Genge v. Wachter (1899) 4 Terr. L.R. 122. 
Re Land Titles Act, 1894, and Blanchard Estate (1901) 5 Terr. L.R. 240.

Sawyer \& Massey v. Waddell (1904) 6 Terr. L.R. 45.

Bocz v. Spiller (1905) 6 Terr. L.R. 225.

Evans v. Postiel (1910) 3 A.L.R. 141.

Re Section 113 Land Titles Act (1913) 6 A.L.R. 111.

Merchants Bank v. Price (1914) 7 A.L.R. 344.

Seay v. Sommerville (1917) 11 A.L.R. 201.

Adanac Oil Co. Ltd. v. Stocks (1916) 11 A.L.R. 214.

Lee v. Armstrong and Harrison (1917) 13 A.L.R. 160.

Robin Hood Mills Ltd. v. Haimson (1918) 14 A.L.R. 196.

Marshall Wells Alberta Company Ltd. v. Alliance Trust Company (1920) 15 A.L.R. 571.

McRoberts v. Registrar of Northern Alberta Land Registry District (1923) 19 A.L.R. 373.

Talboudet v. Junker (1927) 22 A.L.R. 436.

Bocz v. Spiller (1905) 2 W.L.R. 281.

C.P.R. v. Silzer (1910) 14 W.L.R. 274 (Sask.).

Fredericks v. North-West Thresher Co. (1910) 15 W.L.R. 66 (Sask.).

Edmonton Mtge. Co. v. Gross (1911) 18 W.L.R. 385 (Alta.). MacKenzie v. William Gray \& Sons Co. Ltd. (1914) 28 W.L.R. 322 (Sask.).

Union Bank of Canada v. Lumsden Milling Co. (1915) 31 W.L.R. 801 (Sask.).

Adanac Oil Co. v. Stocks (1916) 33 W.L.R. 864 (Alta.).

Quebec Bank v. Royal Bank (1916) 34 W.L.R. 137 (Alta.).

Robinson v. McCauley and Gunn (1913) 5 W.W.R. 789 (Man.).

Merchants Bank v. Price (1914) 5 W.W.R. 1279 (Alta.).

Hart v. Rye (1914) 5 W.W.R. 1280 (Alta.).

Traunweiser v. Johnson; Re Mucklow and Johnson's Contract (1915) 8 W.W.R. 1028 (Alta.).

Foss v. Sterling Loan (1915) 8 W.W.R. 1092 (Sask.).

Union Bank of Canada v. Lumsden Milling Co. (1915) 8 W.W.R. 1167 (Sask.).

Re Land Registry Act; Re Mandeville et al. [1917] 1 W.W.R. 1522 (B.C.).

Weidman v. McClary Mfg. Co. [1917] 2 W.W.R. 210 (Sask.).

Lee v. Harrison [1917] 3 W.W.R. 571 (Alta.).

Re Land Titles Act; Re Beaver Lumber Co. Ltd. [1917] 3 W.W.R. 760 (Sask.).

Grace v. Keubler [1917] 3 W.W.R. 983 (Alta.).

Drewry v. Cowie [1919] 2 W.W.R. 388 (Sask.).

Nichol v. Pedlar \& Johnston [1919] 3 W.W.R. 712 (Sask.).

Marshall Wells v. Alliance Trust Co. [1920] 1 W.W.R. 368 (Alta.). 
In Re Land Titles Act [1921] 3 W.W.R. 427 (Sask.).

Morton and Cowell v. Hoffert [1924] 2 W.W.R. 529 (Alta.).

Weyerhaeuser v. Scott [1924] 2 W.W.R. 605 (Sask.).

In Re Winding-up Act; In Re Land Titles Act; In Re Leitch Collieries Ltd. [1926] 1 W.W.R. 528 (Alta.).

Hay v. McCulloch's Ltd. [1930] 1 W.W.R. 434 (Alta.).

Gregg v. Palmer [1932] 2 W.W.R. 241 (B.C.).

Toole Peet Trust Co. v. London Life Insurance Co. [1935] 3 W.W.R. 311 (Alta.).

In Re Land Registry Act; In Re Twamley [1950] 2 W.W.R. 1259 (B.C.).

In Re Land Registry Act; In Re Application of Penn (1951) 4 W.W.R. (NS) 452 (B.C.).

Re Land Titles Act; Re Lot 20, Block 13, Plan FZ 2265, Regina, Sask. (1959) 30 W.W.R. (NS) 481 (Sask.).

Emerson v. Simpson (1962) 38 W.W.R. (NS) 466 (B.C.).

Re Judgments Act; Regina v. Hamilton (1962) 39 W.W.R. (NS) 545 (Man.).

Re Real Property Act; Dominion Lumber Winnipeg Ltd. v. Winnipeg District Registrar (1963) 41 W.W.R. (NS) 343 (Man.).

Arn Eastham Ltd. v. Samson (1963) 41 W.W.R. (NS) 435 (B.C.).

Oliver Chemical Co. (Lethbridge) Ltd. v. Fisher and Alta. Hail Insurance Board and Royal Bank of Canada (1963) 42 W.W.R. (NS) 269 (Alta.).

Nova Holdings Ltd. v. Western Factors Ltd. and Bird Const. Co. Ltd. (1965) 51 W.W.R. (NS) 385 (Alta.).

Re Parton (1967) 61 W.W.R. (NS) 171 (Alta.).

Re Young Estate (1968) 66 W.W.R. (NS) 193 (B.C.).

Re McDonald (1969) 71 W.W.R. (NS) 444 (B.C.).

Straka v. Straka (1970) 73 W.W.R. (NS) 759 (Alta.).

Sunglo Lumber Ltd. v. McKenna [1974] 5 W.W.R. 572 (B.C.).

Section 129 In Re Brown (1904) 7 Terr. L.R. 67.

Hansen v. Taylor [1933] 2 W.W.R. 16 (B.C.).

Holigrocki (otherwise Holigroski) v. Holigrocki and Registrar of Land Titles (Winnipeg) (1966) 58 W.W.R. (NS) 368 (Man.).

Section 131 C.P.R. Co. v. Mang (1908) 8 W.L.R. 774 (Sask.).

Maitland v. Mathews (1915) 8 W.W.R. 274 (Alta.).

Anderson v. Stasiuk [1927] 1 W.W.R. 49 (Sask.).

Re Land Registry Act; Re Nosalek (1967) 61 W.W.R. (NS) 659 (B.C.).

Section 132 Re Donally Tax Sale (1902) 5 Terr. L.R. 270. 
Section 134 In Re Land Titles Act; In Re A. MacDonald Co. Ltd. and Western Grocers Ltd. [1922] 3 W.W.R. 662 (Sask.).

Section 136 Arnot and Smith v. Peterson (1912) 4 A.L.R. 324.

Rigby v. Rigby (1922) 17 A.L.R. 1.

Re International Harvester Co. of America (1909) 11 W.L.R. 29 (Sask.).

Re MacCullough and Graham (1912) 2 W.W.R. 311 (Alta.).

Re Grand Trunk Pacific Branch Lines Co.'s Caveat (1912) 3 W.W.R. 387 (Sask.).

Thompson v. Yockney (1914) 6 W.W.R. 1397 (Man.).

Imperial Elevator Co. v. Olive (1914) 6 W.W.R. 1562 (Sask.).

Re Registration of a Caveat (1915) 8 W.W.R. 866 (Sask.).

Re C.P.R. Caveat (1916) 10 W.W.R. 10 (Sask.).

In Re Land Titles Act; The Union Supply Company's Case [1918] 2 W.W.R. 305 (Sask.).

Hay v. McCulloch's Ltd. [1930] 1 W.W.R. 434 (Alta.).

Lewis F. Button Ltd. v. Fuglerud [1941] 3 W.W.R. 812 (Sask.).

Croxton v. Racine (1951) 1 W.W.R. (NS) 593 (Alta.).

City of Vancouver v. Registrar of Vancouver Land Registration District (1955) 15 W.W.R. (NS) 351 (B.C.).

C.P.R. Co. v. District Registrar of Dauphin Land Titles Office (1956) 18 W.W.R. (NS) 241 (Man.).

Toronto Dominion Bank v. City of Saskatoon (1967) 60 W.W.R. (NS) 360 (Sask.).

Heiden v. Huck [1971] 5 W.W.R. 446 (Alta.).

Siewert v. Seward [1974] 4 W.W.R. 211 (Alta.).

Section 137 Wanek v. Thors (1928) 23 A.L.R. 602.

Re International Harvester Co. of America (1909) 11 W.L.R. 29 (Sask.).

Re Cass and Canada Traders Ltd. (1910) 15 W.L.R. 194 (Man.).

Merchants Bank of Canada v. Hastie (1914) 27 W.L.R. 764 (Alta.).

Re Land Titles Act; The Holland Canada Mortgage Co. Ltd.'s Case [1918] 3 W.W.R. 345 (Sask.).

Union Bank of Canada v. Turner [1922] 3 W.W.R. 1138 (Man.).

Wilkinson v. Shackleton [1930] 1 W.W.R. 721 (Alta.).

Hamlyn v. Kaplan [1936] 2 W.W.R. 293 (Alta.).

In Re Land Registry Act; In Re Conveyance from Cawley to Lumley [1947] 2 W.W.R. 526 (B.C.).

Rystephaniuk v. Prosken (1951) 3 W.W.R. (NS) 76 (Man.). Ruptash v. Zawick (1956) 2 D.L.R. (2d) 145 (Alta.). 
C.P.R. Co. v. District Registrar of Dauphin Land Titles Office (1956) 18 W.W.R. (NS) 241 (Man.).

Ukrainian Greek Orthodox Church of Canada v. Independent Bnay Abraham Sick Benefit and Free Loan Association and Riverside Cemetery (1959) 28 W.W.R. (NS) 97 (Man.).

Prudential Insurance Company of America v. Junak; Mamczasz v. Bank of Montreal [1975] 4 W.W.R. 522 (Alta.).

Section 141 Re Caveat on Unpatented Lands (1914) 6 W.W.R. 676 (Sask.).

In Re Land Titles Act; Lee's Case [1919] 1 W.W.R. 401 (Sask.).

In Re Land Titles Act; Canada Life Assurance Co.'s Case [1919] 2 W.W.R. 47 (Sask.).

Crown Lumber Co. Ltd. v. Stanolind Oil and Gas Co. Ltd.; Molner v. Stanolind Oil and Gas Co. Ltd. (1957) 21 W.W.R. (NS) 352 (Alta.).

Re Paulette's Application to file a Caveat [1973] 6 W.W.R. 97 (N.W.T.)

Section 142 Pearson v. O'Brien (1912) 1 W.W.R. 1026 (Man.). Rogers Lumber Co. v. Smith (1913) 4 W.W.R. 441 (Sask.). Scott and Cresswell v. Cresswell and Registrar for North Alberta Land Registration District et al. [1975] 3 W.W.R. 193 (Alta.).

Section 144 Re Beufert and Hunter (1913) 26 W.L.R. 405 (Sask.).

Hicks v. Laidlaw (1911) 1 W.W.R. 293 (Man.).

McGreevy v. Murray (1912) 1 W.W.R. 758 (Man.).

Re Removal of Certificate of Lis Pendens (1913) 5 W.W.R. 794 (Sask.).

Re Removal of Lis Pendens (1914) 6 W.W.R. 570 (Sask.).

Forrester v. Lafontaine (1914) 6 W.W.R. 575 (Sask.).

Granby Consolidated Mining, etc. Co. Ltd. v. Esquimalt and Nanaimo Railway Co. [1919] 3 W.W.R. 331 (P.C.).

Radinowsky v. Sheps [1921] 1 W.W.R. 693 (Man.).

Winnipeg Paint and Glass Co. v. Lackman [1923] 3 W.W.R. 361 (Man.).

Phillips v. Greenberg [1924] 3 W.W.R. 160 (Man.).

Zwick v. Rural Municipality of Parkdale [1934] 1 W.W.R. (Sask.).

In Re Land Titles Act; In Re Aylwin and Spencer [1945] 3 W.W.R. 302 (Sask.).

In Re Land Titles Act; In Re Plan No. P. 1652 [1945] 3 W.W.R. 416 (Sask.).

In Re Land Registry Act; Vallstrom v. Staff [1950] 1 W.W.R. 1086 (B.C.). 
Trans-Western Oils Ltd. v. Eckert (1951) 1 W.W.R. (NS) 657 (Alta.).

Rudland v. Romilly (1958) 26 W.W.R. (NS) 193 (B.C.).

Fraser River Ventures Ltd. v. Yewdall; R. A. Nelson Construction Ltd. v. Yewdall (1959) 27 W.W.R. (NS) 368 (B.C.).

Closson and Closson v. Howson (1962) 41 W.W.R. (NS) 275 (Sask.).

Re Land Titles Act; Freeholders Oil Co. Ltd. v. Runge (1963) 41 W.W.R. (NS) 433 (Sask.).

Sheridan v. Warkentin (1963) 42 W.W.R. (NS) 427 (Man.).

Boicey v. Boicey (1964) 47 W.W.R. (NS) 446 (B.C.).

Thiessen Enterprises Ltd. v. Black \& Armstrong and Sovereign Life Assurance Co. of Canada (1967) 58 W.W.R. (NS) 695 (Man.).

Re Land Registry Act; Re Lot 43 New Westminster (1967) 61 W.W.R. (NS) 65 (B.C.).

Nazaruk and Nazaruk v. Registrar, Humboldt Land Titles Office (1969) 71 W.W.R. (NS) 136 (Sask.).

Hugh M. Fraser Ltd. v. Midburn Holdings Ltd. [1971] 2 W.W.R. 387 (B.C.).

Section 146 Sawyer-Massey Co. v. Dennis et al. (1907) 1 A.L.R. 125.

Re Babbit and Boileau (1907) 6 W.L.R. 260 (N.W. Provinces-Calgary).

Re Riddock and Chadwicks Contract (1907) 6 W.L.R. 360 (N.W. Provinces-Calgary).

Section 149 Lundy and McLeod v. Powell [1922] 3 W.W.R. 991 (Sask.). Fayad and Fayad v. Pacula and Pacula [1974] 2 W.W.R. 446 (Alta.).

Section 150 Alloway v. Rural Municipality of St. Andrews (1905) 1 W.L.R. 407 (Man.).

Re Trudge Pork Packing Co. (1908) 7 W.L.R. 507 (Sask.).

Section 152 The Quebec Bank v. The Royal Bank (1916) 9 A.L.R. 435. Brooksbank v. Burn (1910) 15 W.L.R. 661 (Alta.).

Coast Lumber Co. Ltd. v. McLeod (1914) 29 W.L.R. 357 (Sask.).

Alexander v. McKillop and Benjafield (1921) 1 W.W.R. 871 (Alta.).

Arnot v. Peterson (1912) 2 W.W.R. 1 (Alta.).

Stephens v. Bannan and Gray (1913) 5 W.W.R. 201 (Alta.).

Muller v. Schwalbe; Re Royal Bank of Canada and La Banque D'Hochelaga (1914) 7 W.W.R. 817 (Alta.).

McKay v. McDougall [1921] 3 W.W.R. 833 (Sask.).

Bishop v. Western Trust Co. [1922] 3 W.W.R. 818 (Sask.). 
Union Bank of Canada v. Turner [1922] 3 W.W.R. 1138 (Man.).

Nicholas v. Ruf [1936] 3 W.W.R. 647 (Sask.).

Gas Exploration Co. of Alta. Ltd. and Lee v. Cugnet (1954) 12 W.W.R. (NS) 177 (Sask.).

Ukrainian Greek Orthodox Church of Canada v. Independent Bnay Abraham Sick Benefit and Free Loan Association and Riverside Cemetery (1959) 28 W.W.R. (NS) 97 (Man.).

T. M. Ball Lumber Co. Ltd. v. Zirtz and St. Mary's Parish Credit Union Ltd. (1961) 34 W.W.R. (NS) 625 (Sask.).

Hughes v. Gidosh [1971] 1 W.W.R. 641 (Alta.).

Imperial Oil Ltd. v. Toronto-Dominion Realty Co. Ltd. [1972] 2 W.W.R. 334 (Man.).

Calford Properties Ltd. v. Zeller's (Western) Ltd. [1972] 5 W.W.R. 714 (Alta.).

Bensette and Campbell v. Reece (1973) 2 W.W.R. 497 (Sask.).

Section 155 Hamilton v. Stokes [1921] 2 W.W.R. 921 (B.C.).

Section 157 Spence v. Arnold (1901) 5 Terr. L.R. 176.

Atlantic Realty Co. v. Jackson (1913) 26 W.L.R. 15 (B.C.).

Armstrong v. Marshall (1915) 8 W.W.R. 300 (Alta.).

Renkel v. Neufeld and Neufeld [1929] 3 W.W.R. 111 (Sask.).

In Re Land Titles Act; McAvoy v. Royal Bank of Canada and Interior Trust Co. [1933] 3 W.W.R. 433 (Sask.).

Re Land Registry Act; Re Clary and Hassell Developments Ltd.'s Registration Application (1963) 45 W.W.R. (NS) 389 (B.C.).

Section 160 Schwanda v. Schwanda [1943] 3 W.W.R. 404 (Man.).

Section 161 In Re Land Registry Act; Re Royal Trust Co. (1912) 2 W.W.R. 867 (B.C.).

Re Registration of a Transfer of Mortgage (1915) 9 W.W.R. 491 (Sask.).

In Re Registration of Transfer Law to Zaczkowski [1920] 2 W.W.R. 82 (Alta.).

Canadian American Trust Co. Ltd. and Central Properties Ltd. v. McMullen [1929] 2 W.W.R. 295 (Alta.).

In Re Wallbridge and Registrar of Land Titles [1930] 3 W.W.R. 259 (Alta.).

Registrar of N. Alta. Land Registration District v. Northern Agency Ltd. [1938] 1 W.W.R. 561 (Alta.).

In Re Unearned Increment Tax Act and Land Titles Act (1952) 6 W.W.R. (NS) 657 (Alta.). 
Section 165 Sievell v. Haultain (1911) 18 W.L.R. 388 (Sask.).

Re Bank of Hamilton and McAllister (1912) 22 W.L.R. 849 (Alta.).

Nicholson v. Drew and Norton (1912) 2 W.W.R. 295 (Sask.).

Canada Life Assurance Co. v. Registrar of Assiniboia Land Registration District (1912) 2 W.W.R. 522 (Sask.).

Burden v. Registrar of Land Titles for N. Alberta Land Registration District (1913) 5 W.W.R. 122 (Alta.).

Setter v. The Registrar (1914) 7 W.W.R. 901 (Alta.).

Borbridge v. Borland (1915) 8 W.W.R. 1151 (Sask.).

In Re Land Titles Act; British Western Securities Ltd. Petition [1920] 2 W.W.R. 386 (Sask.).

Ficke v. Spence and Olson [1922] 1 W.W.R. 1271 (Sask.).

McRoberts v. Registrar of N. Alta. Land Registration District [1923] 2 W.W.R. 306 (Alta.).

Lee Mong Kow v. Registrar-General of Titles [1923] 2 W.W.R. 545 (B.C.).

Teel v. Forbes [1924] 2 W.W.R. 996 (Alta.).

White v. Municipal District of Inga and Pidgeon [1929] 1 W.W.R. 172 (Alta.).

Bonneau v. District Registrar of Winnipeg [1935] 3 W.W.R. 181 (Man.).

F. C. Richert Co. Ltd. v. Registrar of Land Titles for S. Alta. Land Registration District [1937] 3 W.W.R. 632 (Alta.).

Gordon v. Hipwell and A.G. of British Columbia (1952) 5 W.W.R. (NS) 433 (B.C.).

McInnis v. District Registrar (1952) 7 W.W.R. (NS) 29 (Man.).

C.P.R. v. Turta (1954) 12 W.W.R. (NS) 97 (Alta.).

Re Land Titles Act; Morris (Executor of Kurp Estate) v. Public Trustee (Wilkins Estate) and Registrar of $N$. Alta. Land Registration District (1958) 26 W.W.R. (NS) 471 (Alta.).

Re Land Registry Act; Heller v. Registrar, Vancouver Land Registration District (1963) 41 W.W.R. (NS) 641 (B.C.).

Holigrocki (otherwise Holigroski) v. Holigrocki and Registrar of Land Titles (Winnipeg) (1966) 58 W.W.R. (NS) 368 (Man.).

Section 167 C.P.R. v. Turta (1954) 12 W.W.R. (NS) 97 (Alta.).

Kaup v. Imperial Oil Ltd. (1962) 37 W.W.R. (NS) 193 (Sask.).

Section 168 Nicholson v. Drew (1912) 21 W.L.R. 189 (Sask.). Teel v. Forbes [1924] 2 W.W.R. 996 (Alta.).

Section 169 R. v. Minister of Finance [1935] 1 W.W.R. 113 (B.C.). 
Section 175 Blackstock v. Registrar of N. Alberta Land Registration District [1917] 2 W.W.R. 938 (Alta.).

Section 176 Bouchard (Administrator of Bouchard Estate) v. Registrar, N. Alta. Land Registration District (1959) 27 W.W.R. (NS) 433 (Alta.).

Section 180 Sutherland v. Rural Municipality of Spruce Grove [1919] 1 W.W.R. 274 (Alta.).

C.P.R. v. Turta (1954) 12 W.W.R. (NS) 97 (Alta.).

Fellger v. Canada Trust Co. (1961) 34 W.W.R. (NS) 418 (Alta.).

Kaup v. Imperial Oil Limited (1962) 37 W.W.R. (NS) 193 (Sask.).

Hudson's Bay Co. v. Shivak (1965) 52 W.W.R. (NS) 695 (Sask.).

Moore v. Moore (1971) 16 D.L.R. (3d) 174 (B.C.).

Section 181 C.P.R. Co. v. District Registrar of Dauphin Land Titles Office (1956) 18 W.W.R. (NS) 241 (Man.).

Coe v. Jones (1970) 73 W.W.R. (NS) 311 (Sask.).

Section 182 In Re Reference by Registrar of Land Titles and In Re Title to Certain Mines and Minerals (1953) 9 W.W.R. (NS) 1 (Alta.).

Section 183 Hiller v. Rural Municipality of Shamrock [1929] 3 W.W.R. 458 (Sask.).

Schiell v. Morrison (1930) 2 W.W.R. 737 (Sask.).

Nova Holdings Ltd. v. Western Factors Ltd. and Bird Const. Co. Ltd. (1965) 51 W.W.R. (NS) 385 (Alta.).

Section 185 Ott v. Lethbridge Brewing and Malting Co. Ltd. (1910) 3 A.L.R. 210.

Re Bank of Hamilton and McAllister (1912) 22 W.L.R. 849 (Alta.).

Re Toohill (1914) 27 W.L.R. 517 (Sask.).

In Re Land Titles Act; British Western Securities Ltd. Petition [1920] 2 W.W.R. 386 (Sask.).

Hansen v. Taylor [1933] 2 W.W.R. 16 (B.C.).

In Re Land Titles Act; In Re Pogue and Lane (1951) 3 W.W.R. (NS) 97 (Alta.).

In Re Land Titles Act and A Certain Certificate of Title (1952) 7 W.W.R. (NS) 21 (Sask.).

In Re S. 70 Land Titles Act (1953) 10 W.W.R. (NS) 68 (Sask.).

C.P.R. v. Turta (1954) 12 W.W.R. (NS) 97 (Alta.).

In Re Appeal by Canadian Gulf Oil Co. from Master of Titles (1955) 14 W.W.R. (NS) 130 (Sask.). 
Shilletto (Executrix of Stahn Estates) and Stahn v. Plitt and Registrar of L.T. (1955) 16 W.W.R. (NS) 55 (Alta.).

Re Certain Mineral Rights (1956) 19 W.W.R. (NS) 646 (Sask.).

Kaup and Kaup v. Imperial Oil Ltd. (1962) 37 W.W.R. (NS) 193 (Alta.).

Duncan v. Joslin (1965) 51 W.W.R. (NS) 346 (Alta.).

Section 188 Re Smith (1908) 8 W.L.R. 131 (Sask.).

Turner v. Clark (1909) 10 W.L.R. 25 (Sask.).

In Re Spencer Estate [1947] 1 W.W.R. 486 (Sask.).

In Re Ray Estate (1952) 6 W.W.R. (NS) 282 (Sask.).

In $\operatorname{Re} S$. 70, Land Titles Act (1953) 10 W.W.R. (NS) 68 (Sask.).

In Re S. 82, Land Titles Act (Ralph Estate) (1954) 12 W.W.R. (NS) 245 (Sask.).

In Re Appeal by Canadian Gulf Oil Co. from Master of Titles (1955) 14 W.W.R. (NS) 130 (Sask.).

Re S. 82, Land Titles Act (Re Schmidt) (1956) 19 W.W.R. (NS) 620 (Sask.).

Re Land Titles Act; Morris (Executor of Kurp Estate) v. Public Trustee (Wilkins Estate) and Registrar of $N$. Alta. Land Registration District (1958) 26 W.W.R. (NS) 471 (Alta.).

Klischies v. Klischies (1959) 30 W.W.R. (NS) 115 (Man.). Turner v. Waterman (1965) 53 W.W.R. (NS) 595 (Sask.).

Rich v. Krause [1975] 1 W.W.R. 87 (B.C.).

Section 191 In Re Land Titles Act; In Re Fielding and North of Scotland Mtge. Co. [1927] 2 W.W.R. 423 (Alta.).

Section 197 McInnis v. District Registrar (1952) 7 W.W.R. (NS) 29 (Man.).

In Re Reference by Registrar of Land Titles and In Re Title to Certain Mines and Minerals (1953) 9 W.W.R. (NS) 1 (Alta.).

Section 198 Re Trustee Act; Re Real Property Act; Re Rules of Court; Re Moir's Estate and Will (1961) 36 W.W.R. (NS) 83 (Man.).

Section 202 Re Mineral Act; Boicey v. McDiarmid (1961) 35 W.W.R. (NS) 670 (B.C.).

Section 203 Allen v. Riopel (1913) 7 A.L.R. 65.

Ross v. Stovall (1919) 14 A.L.R. 334.

In Re Church (1923) 3 W.W.R. 405 (Alta.).

Pelletier v. Municipal District of Opal (1925) 21 A.L.R. 1. 
Wanek v. Thors (1928) 23 A.L.R. 602.

Hooper v. Smith (1905) 2 W.L.R. 195 (N.W.T.).

Independent Lumber Co. v. Gardiner (1910) 13 W.L.R. 548 (Sask.).

Robinson v. Ford (No. 1) (1913) 25 W.L.R. 669 (Sask.).

Shetler v. Foshay and Schmidt (1915) 31 W.L.R. 181 (Sask.).

Cloutier v. Loiselle (1915) 33 W.L.R. 111 (Sask.).

Coventry v. Annable (1912) 2 W.W.R. 816 (Sask.).

Fialkowski v. Fiakowski and Traders Bank of Canada (1911) 1 W.W.R. 216 (Alta.).

Sydie v. Saskatchewan and Battle River Land and Development Co. (1913) 5 W.W.R. 194 (Alta.).

Re Adams and McFarland (1914) 6 W.W.R. 1076 (Alta.).

Brown v. Broughton (1915) 8 W.W.R. 889 (Man.).

Boulter-Waugh \& Co. Ltd. v. Union Bank of Canada ]1919] 1 W.W.R. 1046 (Sask.).

Dominion Fire-Brick \& Clay Products Ltd. v. Pollock [1919] 2 W.W.R. 245 (Sask.).

Ruthenian Greek Catholic Church v. Fetsyk [1922] 3 W.W.R. 872 (Man.).

Watson v. Ogilvie [1924] 1 W.W.R. 837 (Sask.).

Imperial Bank of Canada v. Esakin [1924] 2 W.W.R. 33 (Sask.).

Friesen v. Great West Permanent Loan Co.; Mennonite Land Sales Co. Ltd. v. Friesen [1924] 3 W.W.R. 883 (Sask.).

Waimiha Sawmilling Co. Ltd. v. Waione Timber Co. Ltd. [1925] 3 W.W.R. 95 (N.Z.).

Security Lumber Co. Ltd. v. Lyons [1930] 1 W.W.R. 709 (Sask.).

Security Lumber Co. Ltd. v. Acme Plumbing Shop Ltd. [1930] 2 W.W.R. 663 (Sask.).

Beaver Lumber Co. Ltd. v. Pritchard [1933] 3 W.W.R. 35 (Man.).

In Re F. C. Richert Co. Ltd. [1935] 1 W.W.R. 345 (Alta.).

Hackworth v. Baker [1936] 1 W.W.R. 321 (Sask.).

Dallas v. Toronto General Trusts Corp. [1936] 3 W.W.R. 219 (Man.).

A.G. for British Columbia v. Parker [1937] 3 W.W.R. 705 (B.C.).

Shorey v. L. and District Registrar of the Land Titles District of Winnipeg [1938] 2 W.W.R. 346 (Man.).

Minchau and Minchau v. Busse and Busse [1938] 3 W.W.R. 675 (Alta.).

de Lichtbuer v. Dupmeier [1941] 3 W.W.R. 64 (Sask.).

Davidson v. Davidson [1945] 2 W.W.R. 576 (B.C.).

Pich v. Pich [1947] 1 W.W.R. 428 (Alta.). 
Clark v. Barrick [1949] 2 W.W.R. 1009 (Sask.).

Pfeifer v. Pfeifer [1950] 2 W.W.R. 1227 (Sask.).

C.P.R. v. Turta (1954) 12 W.W.R. (NS) 97 (Alta.).

Canadian Superior Oil of California Ltd. v. Cugnet (1954) 12 W.W.R. (NS) 174 (Sask.).

Zawick v. Ruptash and Lumsden and Zawick (1955) 15 W.W.R. (NS) 518 (Alta.).

Ukrainian Greek Orthodox Church of Canada v. Independent Bnay Abraham Sick Benefit and Free Loan Association and Riverside Cemetery (1959) 28 W.W.R. (NS) 97 (Man.).

Gillson v. Gillson (1961) 36 W.W.R. (NS) 480 (B.C.).

Kaup v. Imperial Oil Limited (1962) 37 W.W.R. (NS) 193 (Sask.).

Credit Foncier Franco-Canadien v. Bennett and Allen and A.G. for British Columbia (1964) 47 W.W.R. (NS) 369 (B.C.).

Zbryski v. City of Calgary (1965) 51 D.L.R. 54 (Alta.).

Re Pacific United Developers (1962) Ltd. (1965) 51 D.L.R. 93 (B.C.).

Protective Holdings Ltd. v. M \& P Transport Ltd. (1969) 70 W.W.R. (NS) 591 (Alta.).

Re Saville Row Properties Ltd. (1969) 7 D.L.R. (3d) 644 (B.C.).

Redi-Mix Concrete Ltd. v. Government of Saskatchewan [1971] 5 W.W.R. 306 (Sask.).

Maurice Demers Transport Ltd. and Demers v. Fountain Tire Distributors (Edmonton) Ltd. [1974] 1 W.W.R. 348 (Alta.).

Section 205 Re Roueche (1907) 7 W.L.R. 278 (Alta.).

Section 345 Re Land Titles Act; The Holland Canada Mortgage Co. Ltd.'s Case [1918] 3 W.W.R. 345 (Sask.).

Section 392 Horne v. Galt (1908) 1 A.L.R. 392.

Boundaries Flewelling v. Johnston (1921) 16 A.L.R. 409.

Bruce v. Canadian Pacific Railway Company (1928) 23 A.L.R. 595.

Oleson v. Jonasson (1906) 3 W.L.R. 466 (Man.).

Cain v. Copeland [1922] 2 W.W.R. 1025 (Sask.).

Rural Municipality of Portage La Prairie v. Rural Municipality of Cartier [1924] 3 W.W.R. 244 (Man.).

Kipp v. Simpson [1928] 3 W.W.R. 331 (B.C.).

Kristiansen v. Silverson [1929] 1 W.W.R. 256 (Sask.).

Kristiansen v. Silverson [1929] 3 W.W.R. 322 (Sask.).

Les Soeurs de Misericorde v. Tellier [1932] 2 W.W.R. 357 (Man.). 
Snell v. Sergent [1940] 1 W.W.R. 573 (Sask.).

Rex (in Right of Province of Manitoba) v. Thomas [1948] 2 W.W.R. 444 (Man.).

Muller v. Mamchur (1955) 15 W.W.R. (NS) 469 (Sask.).

City of Vancouver v. Registrar of Titles (Vancouver) (1959) 27 W.W.R. (NS) 46 (B.C.).

Rotter v. Canadian Exploration Ltd. (1960) 33 W.W.R. (NS) 337 (B.C.).

Re Land Registry act; Re Evans Application (1960) 31 W.W.R. (NS) 665 (B.C.).

Hextall v. P. Burns \& Co. Ltd. (1962) 38 W.W.R. (NS) 422 (Alta.).

Re Quieting Titles Act; Re Bulman's Petition (1966) 56 W.W.R. (NS) 225 (B.C.).

Chuckry v. The Queen [1973] 5 W.W.R. 339 (Man.).

Miller v. A.G. for B.C. [1974] 4 W.W.R. 545 (B.C.).

Joint

Tenancy Perks v. Perks [1950] 2 W.W.R. 189 (B.C.).

Stonehouse v. A.G. of British Columbia (1962) 37 W.W.R. (NS) 62 (B.C.).

Re Young Estate (1968) 66 W.W.R. (NS) 193 (B.C.).

Re Land Registry Act; Brook v. Brook (1969) 69 W.W.R. (NS) 310 (B.C.).

Schofield v. Graham (1969) 69 W.W.R. (NS) 332 (Alta.).

Public Trustee v. Mee [1972] 2 W.W.R. 424 (B.C.).

Foort v. Chapman [1973] 4 W.W.R. 461 (B.C.).

Sunglo Lumber Ltd. v. McKenna [1974] 5 W.W.R. 572 (B.C.).

O'Bertos v. O'Bertos [1975] 2 W.W.R. 86 (Sask.).

Reitsma v. Reitsma [1975] 3 W.W.R. 281 (Sask.).

Munroe v. Carlson [1976] 1 W.W.R. 248 (B.C.).

Limitation

Period
Clarke v. Burton and Registrar of N. Alta. Land Registration District (1959) 27 W.W.R. (NS) 352 (Alta.).

Kaup and Kaup v. Imperial Oil Ltd. (1962) 37 W.W.R. (NS) 193 (Alta.). 